\title{
Secretion of curli fibre subunits is mediated by the outer membrane-localized CsgG protein
}

\author{
Lloyd S. Robinson, ${ }^{1 \dagger}$ Elisabeth M. Ashman, ${ }^{2 \dagger}$ \\ Scott J. Hultgren ${ }^{1}$ and Matthew R. Chapman ${ }^{2 \star}$ \\ ${ }^{1}$ Department of Molecular Microbiology and Microbial \\ Pathogenesis, Box 8230, Washington University School of \\ Medicine, 660S. Euclid Ave, St Louis, MO 63110, USA. \\ ${ }^{2}$ Department of Molecular, Cellular and Developmental \\ Biology, University of Michigan, 830 North University, Ann \\ Arbor, MI 48109, USA.
}

\section{Summary}

Produced by many Enterobacteriaceae spp., curli are biologically important amyloid fibres that have been associated with biofilm formation, host cell adhesion and invasion, and immune system activation. CsgA is the major fibre subunit and CsgE, CsgF and CsgG are non-structural proteins involved in curli biogenesis. We have characterized the role of CsgG in curli subunit secretion across the outer membrane. Directed mutagenesis of CsgG confirmed that its activity is dependent on localization to the outer membrane. Rotary Shadow electron microscopy of purified CsgG suggested that this protein assembles into an oligomeric complex with an apparent central pore. Oligomeric CsgG complexes were confirmed using copurification experiments. Antibiotic sensitivity assays demonstrated that overexpression of CsgG rendered Escherichia coli susceptible to the antibiotic erythromycin. A 22-amino-acid sequence at the $\mathrm{N}$-terminus of CsgA was sufficient to direct heterologous proteins to the CsgG secretion apparatus. Finally, we determined that CsgG participates in an outer membrane complex with two other curli assembly proteins, CsgE and CsgF.

\section{Introduction}

Bacteria use a variety of extracellular fibres to mediate interactions with other cells and with their environment. Assembly of these fibres is complex and often includes chaperon proteins and outer membrane usher-like proteins that are dedicated to the secretion and proper local-

Accepted 10 November, 2005. *For correspondence. E-mail chapmanm@umich.edu; Tel. (+1) 734764 7592; Fax (+1) 734647 0884. ${ }^{\top}$ These two authors contributed equally to this work.

(C) 2005 The Authors

Journal compilation @ 2005 Blackwell Publishing Ltd ization of the fibre subunit proteins. Curli represent a class of thin $(6-8 \mathrm{~nm})$, highly aggregated surface fibres that are part of a complex extracellular matrix promoting biofilm and other community behaviours in Escherichia coli (Zogaj et al., 2001; 2003). Curli also confer binding to fibronectin, laminin, plasminogen and human contact phase proteins (Olsen et al., 1989; Sjobring et al., 1994; Ben Nasr et al., 1996). Among bacterially produced fibres, curli are distinguished by their unusual resistance to chemical and thermal denaturation and by their ability to bind the dyes Congo red (CR) and thioflavin T (ThT). These are properties shared by a growing number of eukaryotic fibres collectively know as amyloids. Amyloid fibres, or the process of amyloid formation, are proposed to cause cell and tissue damage associated with many neurodegenerative diseases (Lashuel et al., 2002; Kayed et al., 2003).

Curliated bacteria stain red when grown on plates supplemented with $\mathrm{CR}$, which provides a convenient way to identify genes important for curli production (Collinson et al., 1993). CR binding has been observed in Salmonella enterica, Klebsiella spp., and Escherichia spp., and the genes necessary for curli production have been found in numerous clinically important Enterobacteriaceae that form biofilms (Zogaj et al., 2003). Curli assembly requires the co-ordinated effort of proteins encoded by the $\operatorname{csg} B A$ and $\operatorname{csg} D E F G$ operons. The $\operatorname{csg} B A$ operon encodes two homologous proteins (CsgA and $\mathrm{CsgB}$ ) that are secreted into the extracellular environment (Hammar et al., 1995; 1996). At the cell surface, CsgA is assembled into a stable CR-binding amyloid fibre in the presence of CsgB. In the absence of the CsgB nucleator, CsgA is secreted from the cell in a soluble, unassembled state. This soluble CsgA can polymerize into curli fibres if it contacts an adjacent cell expressing the CsgB nucleator (and not expressing CsgA) by interbacterial complementation (Hammar et al., 1996; Bian and Normark, 1997).

The csgDEFG operon encodes CsgD, a transcriptional activator of curli synthesis, and three putative assembly factors, CsgE, CsgF and CsgG (Hammar et al., 1995; Romling et al., 1998a). Efficient curli assembly requires the $\mathrm{CsgE}, \mathrm{Csg} F$ and $\mathrm{CsgG}$ proteins. CsgG is a lipoprotein localized to the periplasmic side of the outer membrane (Loferer et al., 1997). In the absence of CsgG, CsgA and CsgB are unstable and curli assembly does not occur. At least three models 
exist to explain these observations. The first suggests that CsgG stabilizes curli subunits in the periplasm, allowing them to be secreted by an unidentified secretion apparatus. The second posits that CsgG assembles into a pore that shuttles curli subunits across the outer membrane. A final model suggests that CsgG stabilizes the subunits in the periplasm and is directly involved in their secretion. The last two models assume that curli subunit stability is coupled to their secretion across the outer membrane.

Secretins are a well-characterized class of bacterial outer membrane proteins thought to function as secretion pores. Secretins often require accessory lipoproteins called pilot proteins for their oligomerization and/or outer membrane localization (Hardie et al., 1996). Lipoproteins have also been implicated directly in the secretion step perhaps as the secretion pore (Schmidt et al., 2001; Bose and Taylor, 2005). Interestingly, while secretins have marked sequence similarity within their C-terminal domains, the lipoproteins hypothesized to function as secretion pores share no apparent sequence similarity (Brok et al., 1999; Schmidt et al., 2001; Bose and Taylor, 2005). In this study, we have characterized the lipoprotein CsgG and our results are consistent with the model that CsgG forms an outer membrane channel that stabilizes the curli subunit proteins by mediating their translocation across the outer membrane. We also demonstrated that CsgG interacted with two periplasmic proteins, CsgE and CsgF, required for efficient curli assembly in vivo. Collectively, our data suggest a new model of curli biogenesis where CsgG is the point of convergence in a pathway enabling curlin subunit secretion.

\section{Results}

The role of CsgG in curlin secretion was investigated. CsgG is an outer membrane-localized lipoprotein required for curli production and for CsgA and CsgB stability (Loferer et al., 1997). A convenient measure of subunit secretion is the ability of a cell to act as a CsgA donor or acceptor during interbacterial complementation (Hammar et al., 1995; Chapman et al., 2002). For example, a csgBor $c s g F^{-}$strain will secrete soluble CsgA to the cell surface that can be polymerized on the surface of a $\mathrm{CsgB}^{+}$acceptor strain. As a csgG strain does not assemble curli, interbacterial complementation was used to assess its ability to produce functional CsgA and CsgB proteins. A csgG strain (LSR1) is white when streaked on CR plates. As shown in Fig. 1A, a csgG strain was unable to accept CsgA from a $\operatorname{csg} B^{-}$(MHR261) donor, nor was it able to donate CsgA to a $\operatorname{csg} A^{-}$(MHR204) acceptor strain.

To assess whether CsgG was sufficient for CsgA secretion, CsgA was expressed from a plasmid in the complete $\Delta c s g$ strain, LSR12. In this system, the expression of the
Csg proteins is under the control of IPTG. As shown in Fig. $1 \mathrm{~B}$, accumulation of $\mathrm{CsgA}$ in the supernatant was observed in strains that expressed CsgG (Fig. 1B, lanes 2, 3 and 5). Expression of CsgE did not significantly alter CsgA accumulation or secretion under these conditions. Strains expressing CsgE, CsgF and CsgG from a single operon contained on plasmid pMC5 consistently secreted less CsgA than strains expressing CsgG from pMC1 (Fig. 1B) (Chapman et al., 2002). Notably, CsgE is not required for $\mathrm{CsgA}$ secretion when $\mathrm{CsgG}$ is overexpressed from the IPTG-inducible trc promoter (Fig. 1B), but CsgE is critical for CsgA stability and curli formation when CsgG is expressed at wild-type (WT) levels from the chromosome (Chapman et al., 2002).

The observation that coexpression of CsgA and CsgG is sufficient for CsgA secretion suggests that these proteins interact. CsgA can be divided into at least three identifiable domains: the N-terminal Sec-dependent signal sequence, the first 22 amino acids of the mature protein, and a C-terminal domain that is predicted to form the amyloid core of the fibre (Collinson et al., 1999). The $\mathrm{N}$ terminal 22 amino acids of the mature CsgA protein do not constitute an integral part of the fibre (Collinson et al., 1999), but are required for CsgA stability and, possibly, secretion (L. Robinson, unpubl. results). To assess whether the mature $\mathrm{N}$-terminal 22 amino acids of CsgA are sufficient for interaction with $\mathrm{CsgG}$, the N-terminal 42 amino acids of premature CsgA (including the CsgA Secdependent signal sequence and the first 22 amino acids of the mature protein) were fused to the mature PhoA protein (Fig. 1C). This fusion protein was expressed from the araBAD promoter in plasmid pAph2. A second PhoA construct was made that lacked the $22 \mathrm{~N}$-terminal residues of CsgA, called pAph1 (Fig. 1C). A C-terminal HA epitope tag on PhoA allowed for the immunoprecipitation of this protein using $\alpha-\mathrm{HA}$ antibodies. Six C-terminal histidine residues were added to $\mathrm{CsgG}$ and this fusion construct was cloned behind the trc promoter in pTRC99A creating pMC2. This plasmid was able to complement CRbinding and curli formation to a csgG null mutant (M.R. Chapman, unpubl. data). This construct also drove CsgG expression in cells growing logarithmically in Luria-Bertani (LB) media - a condition where expression from $\operatorname{csgBA}$ and $\operatorname{csg} D E F G$ promoters is undetectable (Romling et al., 1998b). Loferer et al. (1997) reported that when CsgG was expressed from its native promoter it fractionated exclusively to the Triton-X100 insoluble outer membrane fraction. We have confirmed these results and we have also determined that, when total membranes are treated with sarkosyl, natively expressed CsgG is found almost entirely in the sarkosyl insoluble outer membrane fraction (data not shown). When CsgG is overexpressed from the trc promoter, significant amounts are found in both sarkosyl soluble and insoluble fractions (Fig. 1D, 

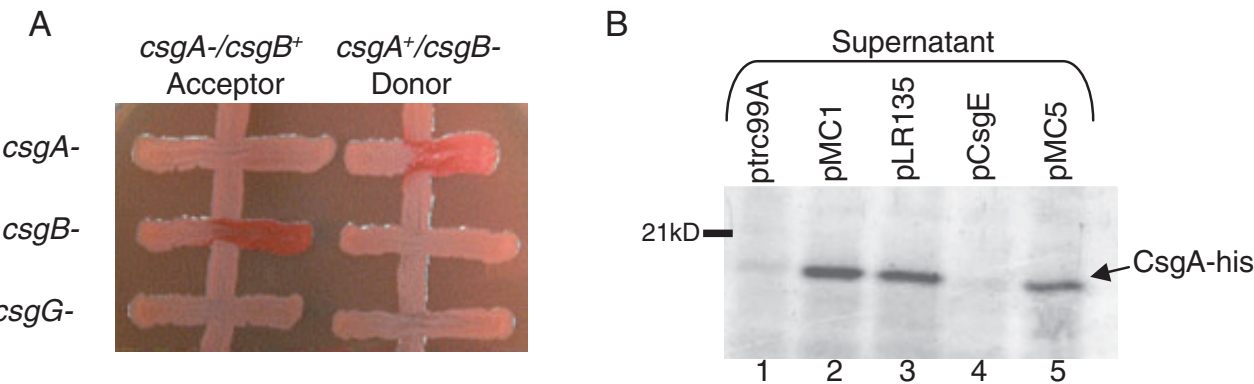

C Aph2

* ${ }^{*}$ VVPQYGGGGGNHGGGGNNSGPVTKARTPEMPVLENRAAQGDIT...

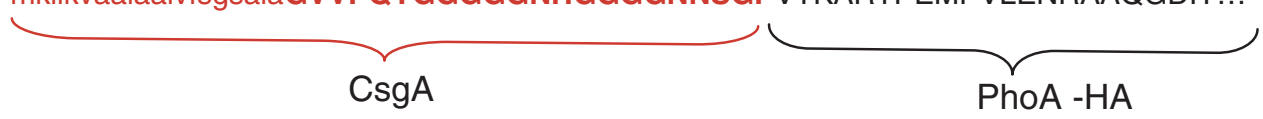

Aph1

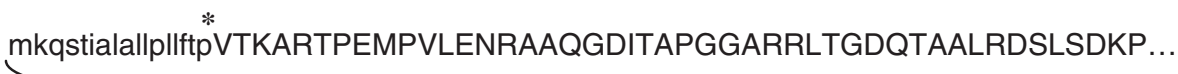
PhoA -HA

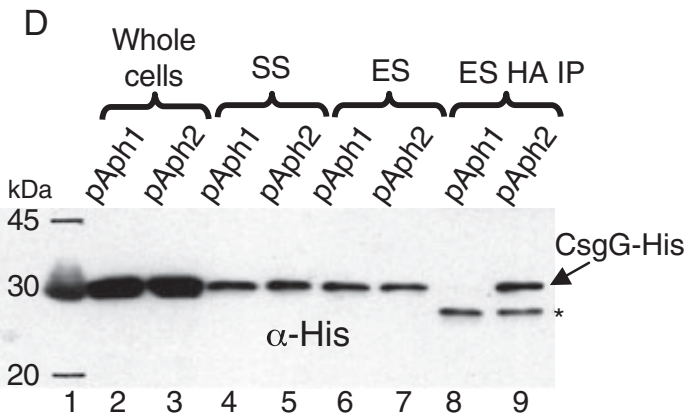

Fig. 1. CsgG is required for interbacterial complementation and CsgA secretion.

A. Interbacterial complementation and CR binding of $\operatorname{csg} G^{-}$mutants. The $\operatorname{csg} B^{+}$acceptor strain MHR204 and the $\operatorname{csg} A^{+}$donor strain MHR261 were streaked from the top of the plate to the bottom. The horizontal cross-streaks were made from left to right with the indicated strains. B. Expression and batch purification of CsgA-his. Supernatants from cells containing pMC3 $\left(\operatorname{csg} A^{+}\right)$and the indicated plasmids were collected and mixed with $20 \mu \mathrm{l}$ of Ni-NTA beads. After incubation for $20 \mathrm{~min}$ at room temperature with gentle rocking, samples were briefly centrifuged to pellet beads, decanted and resuspended in $1 \times$ SDS loading buffer. CsgA-his migrated with an apparent molecular mass of approximately $17 \mathrm{kDa}$ and is indicated with an arrow.

C. N-terminal sequences of WT PhoA (Aph1) and the CsgA-PhoA fusion protein (Aph2). An asterisk indicates the predicted signal peptidase II cleavage site.

D. Co-immunoprecipitation of Aph2 and CsgG. CsgG-his was detected in cell fractions prepared from cells expressing Aph2 (lanes 3, 5, 7 and 9) or Aph1 (lanes 2, 4, 6 and 8) as described in the Experimental procedures by Western blot with $\alpha$-His antibodies. Samples that were immunoprecipitated (IP) from the Elugent soluble (ES) fraction with $\alpha$-HA antibodies were loaded into lanes 8 and 9 . The sarkosyl soluble (SS) fraction is also shown (lanes 4 and 5). CsgG-his migrates at approximately $30 \mathrm{kDa}$ and the asterisk indicates the $\alpha$-HA IgG protein recognized by the $\alpha$-His antibody in the immunoprecipitation samples.

lanes 4-7). The sarkosyl soluble protein observed when CsgG is overexpressed may represent CsgG that is associated with the inner membrane or CsgG that is only weakly interacting with the outer membrane at the time of fractionation. LSR12 (C600:: $\Delta c s g)$ containing pMC2 was transformed with either pAph1 or pAph2. We found that CsgG was present in the Elugent soluble material derived from the sarkosyl insoluble fraction when CsgG was expressed with either WT PhoA (Aph1) or with the CsgAPhoA fusion protein (Aph2) (Fig. 1D, lanes 6 and 7). CsgG was specifically immunoprecipitated with $\alpha-\mathrm{HA}$ antibodies only when CsgG and the CsgA-PhoA-HA fusion protein (Aph2) were coexpressed (Fig. 1D, lane 9). CsgG was not immunoprecipitated when coexpressed with WT PhoA-HA (Fig. 1D, lanes 8), suggesting that the $22 \mathrm{~N}$-terminal amino acids of CsgA are sufficient for mediating an interaction between $\mathrm{CsgG}$ and $\mathrm{CsgA}$.

\section{Purification and structural analysis of CsgG}

CsgG-his was expressed from pMC2 and purified using affinity chromatography as described in the Experimental 
procedures. Outer membranes were recovered by detergent extraction and Elugent soluble material was passed over a nickel NTA column. Nickel NTA-purified CsgG migrated near its predicted molecular weight of $29 \mathrm{kDa}$ (Fig. 2A), although a smaller band was consistently observed in elution fractions that contained full-length CsgG (Fig. 2A, lane 6). The lower molecular weight band is apparently $\mathrm{N}$-terminally truncated $\mathrm{CsgG}$, as this band was recognized by $\alpha$-His antibodies (data not shown).

Gram negative outer membrane secretion pores have been resolved by electron microscopy (EM) as 12-20 nm wide barrel-like structures (Thanassi et al., 1998; Brok et al., 1999). Observation of purified CsgG-his by rotary shadowing EM revealed discrete structures of approximately uniform shape and size. The observed structures were $12-15 \mathrm{~nm}$ wide with an apparent central pore of approximately $2 \mathrm{~nm}$ (Fig. 2C). The structures observed in Fig. $2 \mathrm{C}$ suggest that $\mathrm{CsgG}$ forms pore-containing oligomers in the outer membrane. Although these images suggest that CsgG forms an oligomeric structure, rotary replication can result in an enlargement of the imaged objects, which complicates precise estimation of the size of the oligomer complex or pore size (Thanassi et al., 1998). Therefore, we utilized two differently tagged versions of CsgG to biochemically confirm CsgG-CsgG interactions. Strain $\mathrm{C} 600$ containing inducible plasmids that express CsgG-his (pMC2) and CsgG-HA (pLR92) was grown to mid-log phase and induced as described in the Experimental procedures. Outer membrane fractions were collected from strains containing pMC2 alone, pLR92 alone, or pMC2 and pLR92 together. The Elugent soluble material derived from these outer membrane fractions was loaded onto a Ni-NTA column and CsgG-his was immobilized on the column. Proteins were eluted from the column and the eluates were probed with anti-HA antibodies. CsgG-HA was detected in the eluate only when CsgG-his was coexpressed, suggesting that CsgG-his and CsgG-HA formed a complex that was stable throughout Ni-NTA purification (Fig. 2B).

\section{Antibiotic sensitivity assays}

We next ascertained whether CsgG expression modified the permeability of the outer membrane by using antibiotic sensitivity assays. Erythromycin does not normally pass the Gram negative outer membrane because of hydrophobic repulsion forces. Therefore, this bacteriostatic antibiotic can be used to detect the presence of channels in the outer membrane (Augustus et al., 2004). As shown in Fig $3 \mathrm{~A}, 30 \mu \mathrm{g} \mathrm{ml}^{-1}$ erythromycin did not appreciably affect growth of LSR12 containing vector alone. In contrast, LSR12 containing pMC1 displayed severe growth defects in the presence of erythromycin (Fig. 3A). Therefore, it appeared that CsgG expression increased outer membrane permeability, thus allowing erythromycin to enter the cell and poison translation. This is in agreement with our observation that CsgG assembled into pore-like structures with an apparent central cavity of $2 \mathrm{~nm}$, which would be large enough to allow erythromycin to pass. Importantly, bacteria expressing CsgG did not exhibit growth defects in the absence of erythromycin (Fig. 3A), suggesting that CsgG did not grossly affect membrane integrity. Erythromycin sensitivity was also observed when MC4100 cells overexpressing CsgG were grown on YESCA plates (data not shown). Vancomycin is another antibiotic that is often used to gauge membrane permeability because, like erythromycin, it cannot pass the hydrophobic outer membrane (Schmidt et al., 2001). We observed relatively modest growth inhibition using vancomycin, even in cells overexpressing CsgG from pMC1 (Fig. 3B), suggesting that CsgG-dependent erythromycin sensitivity is not due to a general membrane defect and may be due to presence of a CsgG pore in the outer membrane. At twice the size of erythromycin (1440 Da versus $740 \mathrm{Da}$ for erythromycin), we reasoned that vancomycin might not pass through the pore fashioned by CsgG oligomers, although it is possible that chemical differences between the antibiotics are responsible for the exclusion of vancomycin from CsgGexpressing cells.

\section{Lipid modification is required for outer membrane Iocalization}

CsgG has been demonstrated to be a lipoprotein localized to the outer membrane. Consequently, it has been suggested that CsgG is targeted to the outer membrane by the LOL transport system (Loferer et al., 1997). Bacterial lipoproteins are modified on a cysteine residue located at the N-terminus (Narita et al., 2004). CsgG contains a conserved $\mathrm{N}$-terminal cysteine residue that is the putative site of lipidation. We constructed a substitution mutant of this cysteine residue (C16A) and found its expression to be toxic to cells (our unpublished data). Therefore, we removed the CsgG lipoprotein signal sequence, including the $\mathrm{N}$-terminal cysteine, and replaced it with the signal sequence from the PhoA protein (Fig. 4A). The expression of this protein chimera, called CsgGss, was induced in log phase cells and then localized using detergent fractionation. WT CsgG consistently localized with outer membrane fractions, while CsgGss localized predominately with the soluble periplasmic fraction (Fig. 4B). The ability of CsgGss to complement a csgG mutant was tested. No $\mathrm{CR}$ binding was detected in a $\operatorname{csg} G^{-}$mutant, although this strain could be complemented by expression of WT csgG from the trc promoter in pMC1 (Fig. 4C). Partial complementation was achieved when WT $\operatorname{csg} G$ was expressed from the $\operatorname{csg} B A$ promoter in pLR93. In contrast, CsgGss 
A

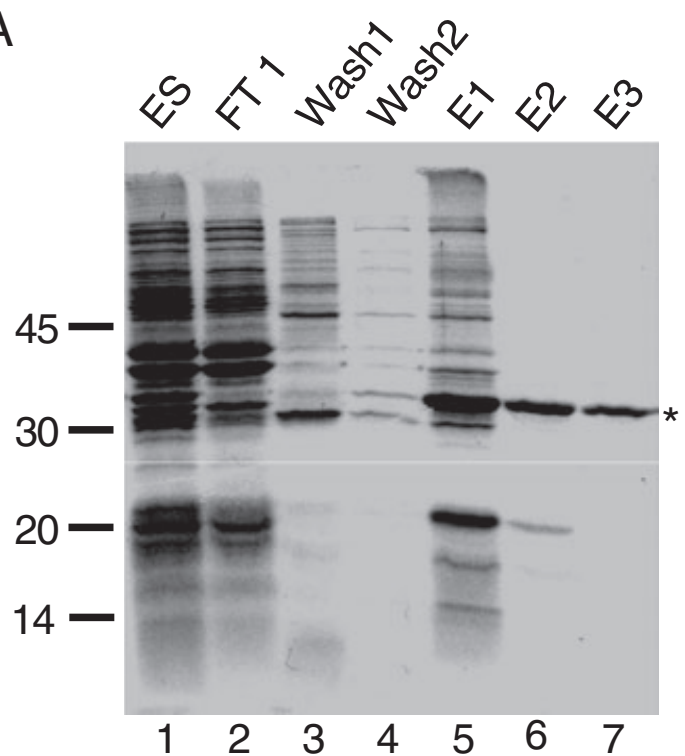

B

$\frac{\begin{array}{l}\text { CsgG-his/ } \\ \text { CsgG-HA }\end{array} \text { ES FT E1 }}{\text { ES FT E1 }} \frac{}{\text { ES FT E1 }}$

$\mathrm{kDa}$

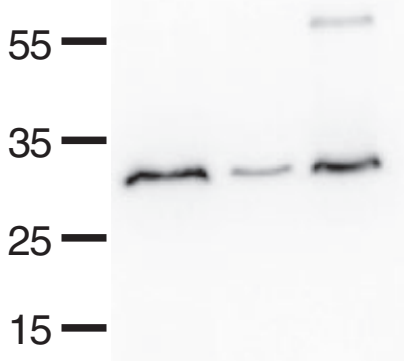

C

$\begin{array}{lllllllll}1 & 2 & 3 & 4 & 5 & 6 & 7 & 8 & 9\end{array}$

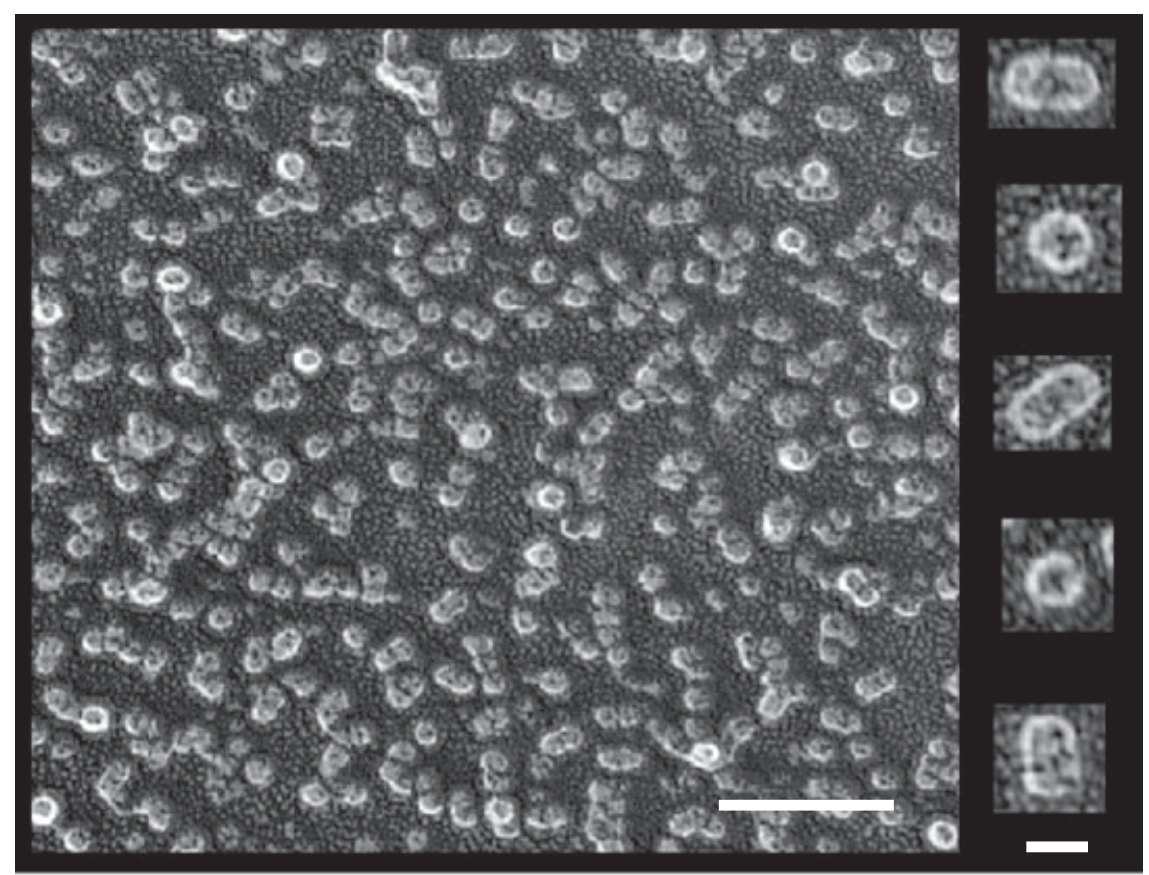

Fig. 2. Purification and structural analysis of CsgG.

A. C600/pMC2 cells were harvested and the Elugent soluble (ES) outer membrane fraction (lane 1) was applied to a Ni-NTA column in five column volumes of HNE and $1 \mathrm{ml}$ flow-through (FT) collected (lane 2). The column was then washed with five column volumes HNE containing $10 \mathrm{mM}$ imidizole. The first (W1) and last (W2) millilitre of this wash was collected for analysis (lanes 3 and 4). CsgG-his bound to the column was eluted with HNE containing $100 \mathrm{mM}$ imidizole and collected in three $1 \mathrm{ml}$ fractions, E1-E3 (lanes 5-7). Fractions were resolved by SDSPAGE and visualized by Coomassie brilliant blue staining. The asterisk at $30 \mathrm{kDa}$ indicates the molecular weight of CsgG.

B. CsgG forms an oligomer in the outer membrane. ES outer membrane fraction from cells expressing CsgG-his (lane 4), CsgG-HA (lane 7), or both (lane 1) were isolated and CsgG-his was purified by Ni-NTA chromatography as described in Experimental procedures. Analysis of the fractions by Western blot analysis with $\alpha-\mathrm{HA}$ antibodies revealed CsgG-HA in the eluate only when CsgG-his was coexpressed (lane 3). The band at $\sim 55 \mathrm{kDa}$ in lane 3 is an unidentified cross-reacting band that is sometimes seen when CsgG is purified from C600.

C. Rotary Shadow EM analysis of CsgG-his protein reveals donut-shaped structures approximately $13 \mathrm{~nm}$ in diameter. Scale bar left panel $=100 \mathrm{~nm}$. Right panel shows enlargements of single particles from the left panel. Scale bar right panel $=15 \mathrm{~nm}$. Rotary shadowing with platinum was performed as described in the Experimental procedures. 

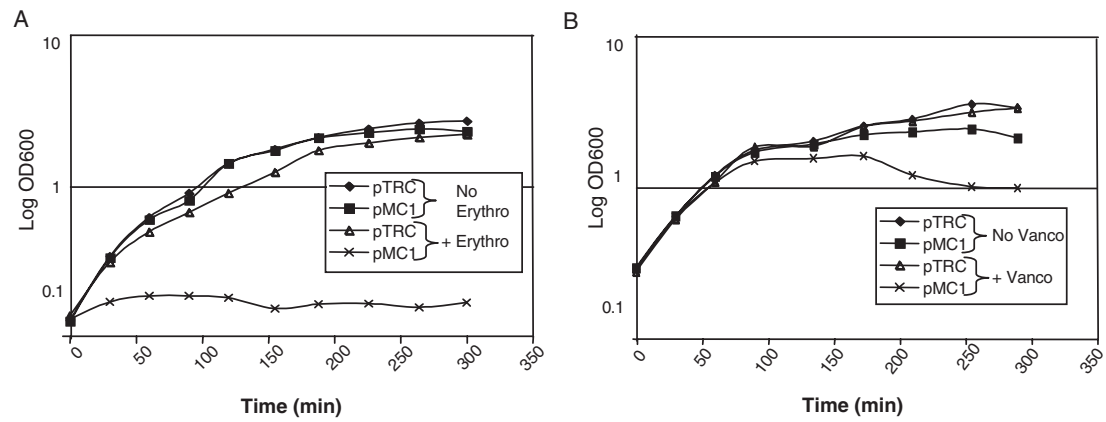

Fig. 3. Antibiotic sensitivity studies.

A. Growth curve of E. coli strain C600 containing plasmid pTRC99A or pMC1 in the presence or absence of $30 \mu \mathrm{gl}^{-1} \mathrm{erythromycin}^{\mathrm{S} \text { Strains }}$ were grown overnight, diluted 1:100 in LB and grown for $30 \mathrm{~min}$ in the presence of $0.05 \mathrm{mM}$ IPTG, before erythromycin was added (time 0). $B$. Growth curve of $E$. coli strain $C 600$ containing plasmid pTRC99A or pMC1 in the presence or absence of $30 \mu \mathrm{gl}^{-1}$ vancomycin. Strains were grown overnight, diluted 1:100 in LB and grown for $30 \mathrm{~min}$ in the presence of $0.05 \mathrm{mg} \mathrm{ml}^{-1}$ IPTG, before vancomycin was added (time 0 ).

was unable to complement the csgG mutant when expressed from either the trc or $\operatorname{csg} B A$ promoters (Fig. 4C). Interestingly, the expression of CsgGss abolished the CR positive phenotype of an otherwise WT strain (Fig. 4C), suggesting that CsgGss is able to interfere with WT CsgG function.

Because the CsgA and CsgB proteins are unstable without CsgG, we tested the ability of CsgGss to stabilize the curli subunits in the absence of WT CsgG. As shown in Fig. 4D, CsgA and CsgB do not accumulate to WT levels when CsgGss is expressed in a csgG- strain, suggesting that proper CsgG localization to the outer membrane is required for the chaperone-like activity of CsgG (Fig. 4D).

\section{Complex with other Csg proteins}

CsgG is expressed from an operon along with three other proteins that are known to play a role in curli formation. $\mathrm{CsgD}$ is a transcriptional activator required for curli gene expression, while CsgE and CsgF are chaperone-like proteins that facilitate curli formation (Chapman et al., 2002; Chirwa and Herrington, 2003; Gerstel et al., 2003). However, the roles of CsgE and CsgF in curli formation are poorly understood. We tested whether CsgE or CsgF could interact with CsgG at the outer membrane. To facilitate this analysis, the CsgE and CsgF proteins were epitope tagged with AU1 or HA respectively. These fusion proteins were able to complement $C R$ binding to $\operatorname{csg} E$ and csgF chromosomal deletion strains (data not shown). C600 with pMC2 and pBAD33 or pLR58 (csgF-HA in pBAD33) was grown and the expression of CsgF-HA and CsgG-his was induced with arabinose and IPTG. Outer membranes were prepared after $1 \mathrm{~h}$ of induction and CsgF-HA was immunoprecipitated with $\alpha-\mathrm{HA}$ antibodies. CsgG was detected in the Elugent soluble outer mem- brane fraction with or without coexpression of CsgF-HA (Fig. 5A, lanes 4 and 5), but was specifically co-immunoprecipitated with $\alpha-\mathrm{HA}$ antibodies only when CsgF-HA was present. This demonstrated that $\mathrm{CsgF}$ and CsgG physically interacted at the outer membrane. C600 containing pMC2 and pBAD33 or pLR169 (csgE-AU1 in pBAD33) was grown to induce CsgG-his and CsgE-AU1 expression. Proteins contained within the sarkosyl soluble inner membrane and Elugent soluble outer membrane fractions were immunoprecipitated with $\alpha$-AU1 antibodies. As shown in Fig. 5B, CsgE-AU1 and CsgG-his were coimmunoprecipitated with $\alpha$-AU1 antibodies from the Elugent soluble fractions. Immunoprecipitation of CsgG-his was completely dependent on expression of CsgE-AU1 (Fig. 5B). CsgG-his was not co-immunoprecipitated with CsgE-AU1 when sarkosyl soluble fractions were used (Fig. 5B, lanes 6-7 and 10-11). These data suggest that CsgG is an integral part of an outer membrane secretion complex that contains at least two other curli assembly proteins, CsgE and CsgF.

\section{Discussion}

Curli biogenesis is a complex process that requires several proteins, including those encoded by the csg operons. The lipoprotein CsgG forms an oligomeric structure in the outer membrane that is required for the secretion of the CsgA and CsgB proteins to the cell surface. The localization of CsgG to the outer membrane is dependent on posttranslational acylation of the cysteine located at the first residue of the mature protein. Overexpression of CsgG renders logarithmically growing cells susceptible to the hydrophobic antibiotic erythromycin, most likely by a specific change in the permeability of the outer membrane. CsgA secretion and stability is dependent on the N-terminal 22 amino acids of CsgA. These 22 amino acids can 
A

\section{WT G: $\quad$ mqrlfllvavmllsg*CLTAPP...}

CsgGss: makqstialallpllftpvtka*ADPLTAPP...

C

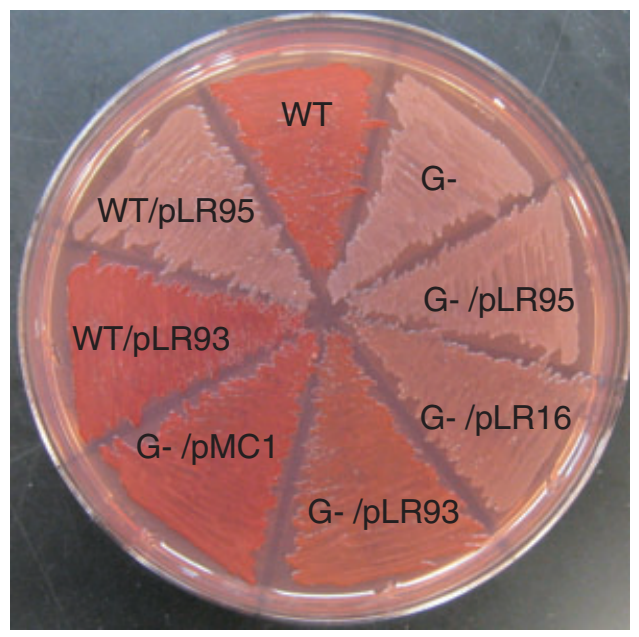

B

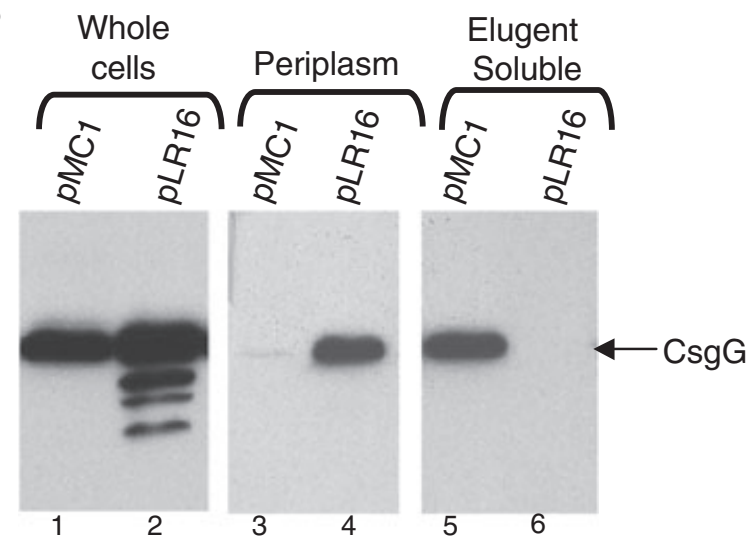

D

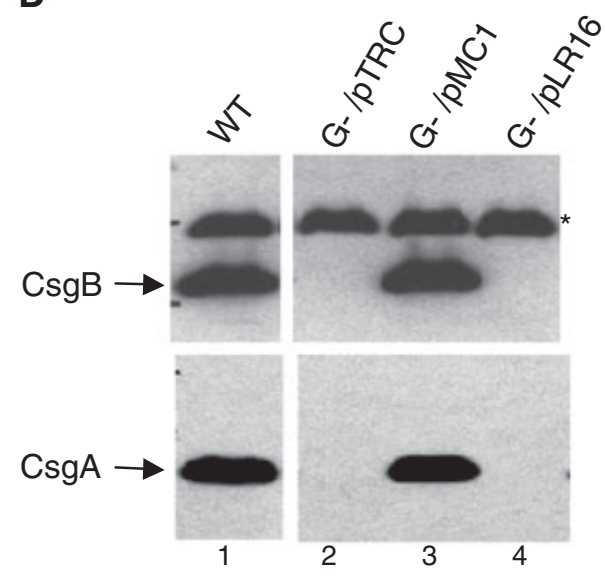

Fig. 4. Lipidation is required for CsgG activity.

A. The N-terminal 21 amino acids of CsgG that encodes a SEC-dependent secretion sequence and lipoprotein modification site are shown. The asterisk indicates the putative signal peptidase II-dependent cleavage site. Shown in red are the N-terminal 25 amino acids from PhoA that were used to replace the lipoprotein signal sequence of CsgG.

B. Expression and localization of CsgG and CsgGss. Cells containing pMC2 or pLR16 were grown to mid-log phase and induced with $0.1 \mathrm{mM}$ IPTG for $2 \mathrm{~h}$ before being harvested and fractionated as described in the Experimental procedures. CsgG and CsgGss were detected using $\alpha-$ His antibodies. Lanes 1 and 2 are whole cell lysates, lanes 3 and 4 are periplasmic fractions and lanes 5 and 6 are Elugent soluble outer membrane fractions. Equal amounts of CsgG-his and CsgGss-his fractions were loaded.

C. $\mathrm{CR}$ binding of $\operatorname{csg} \mathrm{G}$ - strains containing the indicated plasmids after $48 \mathrm{~h}$ of growth at $26^{\circ} \mathrm{C}$ on YESCA plates.

D. Western analysis using $\alpha$-CsgA or $\alpha$-CsgB antibodies. Whole cell lysates of cells grown for $48 \mathrm{~h}$ on YESCA plates were treated with formic acid as described by Chapman et al. (2002) and probed with $\alpha$-CsgA or $\alpha$-CsgB antibodies. The asterisk indicates a non-specific protein recognized by the $\alpha$-CsgB antibody.

also direct non-curli proteins to form a complex with CsgG. CsgE and CsgF also participate in a complex with CsgG, perhaps modifying its secretion activity.

Bacteria use a variety of mechanisms to shuttle proteins to the cell surface, and lipoproteins are often key components of these systems. Lipoproteins that function in protein secretion fall into one of two functionally distinct classes. The first class represents lipoproteins that chaperone secretin proteins to the outer membrane. Pugsley and colleagues have coined the term 'pilot' protein to describe such proteins. The second class of lipoproteins involved in protein secretion is postulated to form an outer membrane pore through which substrate proteins are channelled. Members of several terminal secretion systems predicted to be lipoproteins are directly involved in protein secretion across the outer membrane, including BfpB (Ramer et al., 1996; Schmidt et al., 2001), CfcD (Mundy et al., 2003) and TcpC (Bose and Taylor, 2005). CsgG does not share significant sequence similarity to any of the lipoproteins known to participate in bacterial secretion, yet CsgG homologues are present in many Gram negative bacteria - including many clinically important members of Enterobacteriaceae (Zogaj et al., 2003 and our unpublished data). 
A

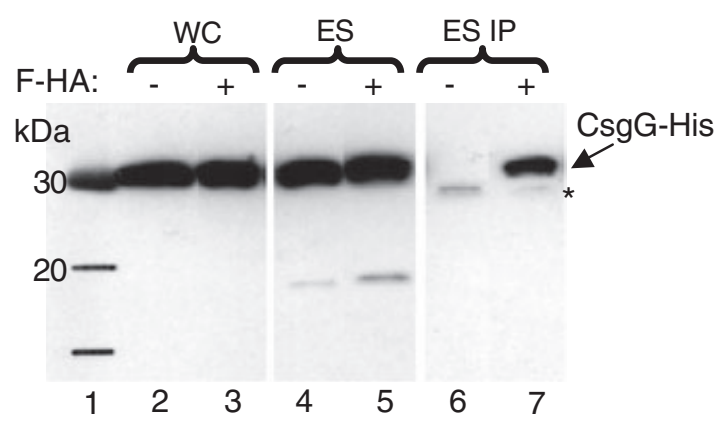

B

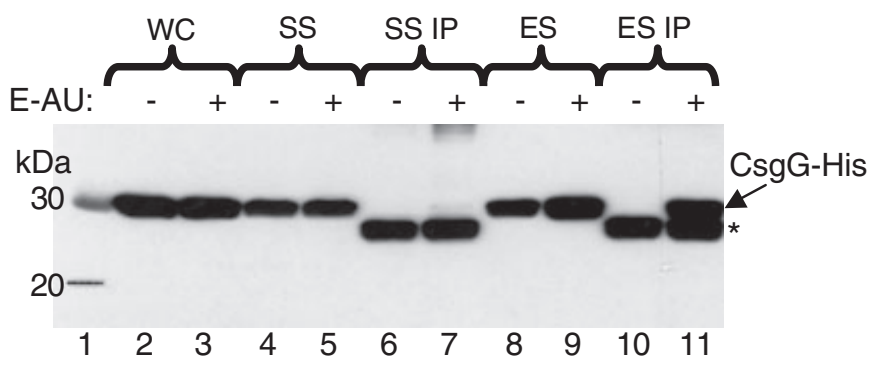

Fig. 5. CsgG interacts with CsgE and CsgF at the outer membrane.

A. Cells expressing CsgG-his and CsgF-HA were fractionated and then immunoprecipitated with $\alpha-\mathrm{HA}$ antibodies where indicated. Samples were blotted and probed with $\alpha$-His antibodies to detect CsgG. CsgG migrates at approximately $30 \mathrm{kDa}$ and is indicated with an arrow.

B. Cells expressing CsgG-his and CsgE-AU1 were fractionated prior to immunoprecipitation with $\alpha$-AU antibodies. CsgG-his migrates at approximately $30 \mathrm{kDa}$ and the asterisk indicates the IgG protein in the immunoprecipitation samples recognized by the $\alpha$-His antibody. WC, whole cells; SS, sarkosyl soluble fraction; ES, Elugent soluble fraction; IP, immunoprecipitation.

In the absence of CsgG, the CsgA and CsgB proteins are not secreted to the cell surface, yet they do not accumulate in the periplasmic space (Loferer et al., 1997). Currently, there are at least two models that explain why CsgA and CsgB are unstable in the absence of CsgG. CsgG might act as a chaperone protein, stabilizing CsgA and $\mathrm{CsgB}$ in the periplasm until they are secreted to the cell surface. Alternatively, CsgG may simply transport CsgA and CsgB to the cell surface where they are not subject to periplasmic proteases. To clarify the mechanism of CsgG, we asked if CsgG localization to the outer membrane was required for CsgA and CsgB stability. CsgG is modified with a palmitate group after secretion across the inner membrane (Loferer et al., 1997). Therefore, the lipidation-specific signal sequence on CsgG was replaced with a general SEC-dependent signal sequence. This protein, called CsgGss, is not lipidated and localizes to the periplasm instead of the outer membrane. Furthermore, CsgGss is unable to restore curli production in a csgG strain (Fig. 4C) and it does not act to stabilize the CsgA or CsgB proteins (Fig. 4D). The failure of CsgGss to stabilize curli subunits cannot be explained by an inability to bind subunits, as CsgGss and the CsgA-PhoA fusion specifically interacted in Far-Western assays (L. Robinson, unpubl. data). This suggests that CsgG must localize to the outer membrane in order to stabilize CsgA and $\mathrm{CsgB}$ and direct their secretion across the outer membrane. Therefore, stability and secretion of CsgA and CsgB appear to be tightly coupled. CsgA and CsgB may be inherently unstable in the periplasm and CsgG-mediated secretion helps to stabilize them by exporting them to the more forgiving extracellular space. The extracellular space is also where curli subunits are proposed to transition into the amyloid state, which might also lead to increased subunit stability (Hammar et al., 1996).
The molecular mechanism behind the dominant negative phenotype observed when CsgGss is expressed along with WT CsgG is unclear. At least two possibilities exist to explain this result. As CsgG forms oligomeric structures in the outer membrane, it is possible that CsgGss can form a non-functional complex with WT CsgG, thereby preventing WT CsgG activity. As shown in Fig. 2, CsgG forms oligomeric structures and it is possible that CsgGss participates in this complex. Alternatively, expression of CsgGss may change the cellular environment such that curli formation is inhibited. For example, if CsgGss is not completely folded then inducible cell stress systems may be activated that negatively affect curli formation. One such system is the Cpx system that can be induced by misfolded proteins in the periplasm (Hung et al., 2001; Nevesinjac and Raivio, 2005; Ruiz and Silhavy, 2005). Not only does the Cpx system upregulate the expression of proteins with protease activity, which might degrade WT CsgG or the curli subunits, but CpxR has also been shown to downregulate the csg operons at the transcriptional level (Prigent-Combaret et al., 2001).

CsgA that has been secreted by CsgG-expressing cells is found in an unstructured state upon purification (Chapman et al., 2002). The size of the structures observed in Fig. $2 \mathrm{C}$ is in agreement with the idea that the curli subunits may be at least partly unfolded during translocation across the outer membrane. However, the mechanism by which CsgA could be maintained in this unfolded state is unclear. CsgG probably does not work alone during curli assembly, despite the discovery that overexpression of CsgG in logarithmically growing cells results in CsgA secretion (Fig. 1B), In this experiment, CsgG and CsgA were expressed from inducible promoters and CsgA was almost exclusively found in the extracellular space. Neither CsgE nor CsgF augmented CsgA secretion when 
CsgG was overexpressed. Yet, CsgE is required for CsgA and CsgB secretion when cells are grown on YESCA plates and the curli proteins are expressed from their native promoters (Chapman et al., 2002). Because CsgE and $\mathrm{CsgF}$ interact with CsgG at the outer membrane, it is possible that under native conditions $\mathrm{CsgE}$ and $\mathrm{CsgF}$ increase the specificity or efficiency of CsgG-mediated secretion. Delineating the molecular details of CsgE and $\mathrm{CsgF}$ function during curli formation will help clarify this unique secretion system.

\section{Experimental procedures}

Plasmids, strains, growth conditions and antibodies

Expression of csg genes at WT levels from MC4100 chromosome or pLR1-derived plasmids was accomplished by growing cells on YESCA agar [ $10 \mathrm{~g}$ Casamino acids, $1 \mathrm{~g}$ yeast extract, $20 \mathrm{~g}$ agar (Fisher, Fairlawn, $\mathrm{NJ}$ ) in $1 \mathrm{l}$ ] at $26^{\circ} \mathrm{C}$ for $48 \mathrm{~h}$. YESCA agar plates supplemented with $10 \mu \mathrm{g} \mathrm{ml}^{-1} \mathrm{CR}$ (Sigma, St. Louis, MO) were used to monitor curli production on colonies. Expression of genes cloned behind the trc promoter in pTRC99A or the ara promoter in PBAD33 was induced by addition of $0.4 \mathrm{mM} \mathrm{IPTG}$ or $0.4 \%$ (w/v) arabinose respectively. Antibiotics were added at the following concentrations as needed: kanomycin $50 \mu \mathrm{g} \mathrm{ml}^{-1}$, ampicillin $100 \mu \mathrm{g} \mathrm{ml}^{-1}$ and chloramphenicol $20 \mathrm{\mu g} \mathrm{ml}^{-1}$. The csgBA promoter was amplified from MC4100 as a Bglll/Pstl fragment using primers LR1F and LR1R (Table S1) and cloned into the BamHI/Pstl sites of pACYC177 to create pLR1. An Ncol site incorporated into the reverse primer LR1R allowed cloning of the Ncol/Pstl fragment of pLomp4 (Table 1) into pLR1, which added BamHI and $\mathrm{Kpnl}$ sites and created pLR2. Western blots were probed as indicated in the Results with the following antibodies: polyclonal antiserum raised against Ni-NTA purified CsgG-his in rabbits by Proteintech Group, Chicago, IL, rabbit polyclonal antibody raised against the CsgB peptide EGSSNRAKIDQTGDY (Sigma, St. Louis, MO), rabbit polyclonal antibody raised against CsgA (Hammar et al., 1996), or commercially available antibodies against commonly used epitopes, as indicated when used.

\section{Purification of CsgG}

CsgG was purified under non-denaturing conditions from strain C600 containing pMC2 and/or pLR92 as indicated in the Results. The strains were grown with aeration in LB broth containing $100 \mu \mathrm{g} \mathrm{ml}^{-1}$ ampicillin and/or $50 \mu \mathrm{g} \mathrm{ml}^{-1}$ kanomycin as appropriate. At $\mathrm{OD}_{600}$ of 0.6-1.0, csgG-his or csgG-HA expression was induced with $0.5 \mathrm{mM}$ IPTG and/or $0.04 \%$ arabinose for $2 \mathrm{~h}$. Bacteria were harvested and resuspended in $20 \mathrm{mM}$ Tris. $\mathrm{HCl}(\mathrm{pH} 8.0)$, lysed with a French press, and outer membrane collected by differential extraction with Sarkosyl (Thanassi and Hultgren, 2000). CsgG was extracted from the sarkosyl insoluble outer membrane fraction by treatment with $0.5 \%$ Elugent (Calbiochem, San Diego, CA). The Elugent soluble fraction was applied to a Ni-NTA column (Qiagen, Chatsworth, CA) in five column volumes of HNE (20 mM Hepes/300 mM NaCl/0.5\% Elugent). The column was washed with five column volumes of $\mathrm{HNE}+10 \mathrm{mM}$ imidazole. An additional wash of five column volumes of $\mathrm{HNE}+20 \mathrm{mM}$ imidizole was performed for the co-purification experiment presented in Fig. 2B. CsgG bound to the column was eluted with HNE containing $100 \mathrm{mM}$ imidazole.

\section{Electron microscopy}

CsgG was purified for inspection by EM analysis as described above with the following modifications: CsgG was extracted from the sarkosyl insoluble outer membrane fraction by treatment with $0.1 \%$ of the non-ionic detergent $n$ dodecyl-beta-D-maltoside (DDM) and the mobile phase of the Ni-NTA column was buffer A $(20 \mathrm{mM}$ Tris. $\mathrm{HCl}$ pH 8.0/ $150 \mathrm{mM} \mathrm{NaCl} / 0.1 \%$ DDM). Purified CsgG was observed by rotary replication as described by Thanassi et al. (1998). Briefly, CsgG purified by Ni-NTA chromatography was adsorbed to mica chips before quick-freezing. These frozen samples were fractured and deep-etched by exposure to a vacuum. Replicas were constructed by rotary shadowing with platinum.

\section{Cell fractionation to determine CsgG and CsgGss localization}

C600 containing pMC1 or pLR16 was grown with aeration in LB broth and addition of $0.5 \mathrm{mM} I P T G$ to the growth media induced csgG or csgGss expression. Cells were harvested and $0.5 \mathrm{~g}$ cell weight divided into two equal portions and either periplasm or membranes were isolated. The periplasmic fraction was obtained by suspending the cells in $20 \mathrm{mM}$ Tris. $\mathrm{HCl}$ $\mathrm{pH} 8.0 / 20 \%$ sucrose/0.5 mM EDTA and $40 \mu \mathrm{l}$ of $25 \times$ Protease Inhibitor Tablets (Roche, Indianapolis, IN) was added before treatment with $75 \mu \mathrm{g} \mathrm{ml}^{-1}$ lysozyme. This suspension was incubated on ice for $40 \mathrm{~min}$ and the reaction was quenched by addition of $1 \mathrm{M} \mathrm{MgCl}_{2}$. Spheroplasts were pelleted by centrifugation at $10000 \mathrm{~g}$ for $20 \mathrm{~min}$ and the supernatant retained for analysis. Membrane fractions were obtained using a modified protocol originally described by Loferer et al. (1997). Briefly, one half of the cells grown as described above were suspended in $20 \mathrm{mM}$ Tris. $\mathrm{HCl} \mathrm{pH} 8.0$ and lysed with a French press before separating the periplasm and cytosol from the total membrane fraction by ultracentrifugation at $100000 \mathrm{~g}$ for $1 \mathrm{~h}$. The inner membrane was then solubilized in $0.5 \%$ sarkosyl and the sarkosyl insoluble outer membrane fraction pelleted by centrifugation at $100000 \mathrm{~g}$ for $1 \mathrm{~h}$. The pellet was suspended in HNE and insoluble material pelleted by centrifugation at $100000 \mathrm{~g}$ for $30 \mathrm{~min}$. The Elugent soluble fraction was retained for analysis. The fractions obtained by these procedures were subjected to SDS-PAGE and the presence of CsgG or CsgGss was detected by immunoblotting with rabbit anti-CsgG antibodies.

\section{Immunoprecipitation}

Elugent soluble outer membrane material was prepared as described above from the strains described in the Results section. One millilitre of the preparation was combined with anti-HA or anti-AU antibody at the concentration recommended by the manufacturer (Covance, Denver, PA) before 
Table 1. Strains and plasmids used in this study.

\begin{tabular}{|c|c|c|}
\hline Strain or plasmid & Relevant characteristics & Source \\
\hline $\mathrm{C} 600$ & F- thr leu thi lac tonA & Campbell (1961) \\
\hline LSR12 & C600:: $\triangle \operatorname{csg} D E F G \Delta \operatorname{csg} B A$ & Chapman et al. (2002) \\
\hline MC4100 & F-araD139 $\Delta($ argF-lac)U169 rspL150(strR) relA1flbB5301 deoC1 ptsF25 rbsR & Casadaban (1976) \\
\hline LSR1 & MC4100csgG::Tn105 & This study \\
\hline MHR204 & MC4100csgA::Tn105 & Hammar et al. (1996) \\
\hline LSR10 & MC4100:: $\Delta \operatorname{csg} A$ & Chapman et al. (2002) \\
\hline MHR261 & MC4100:: $\Delta \operatorname{csg} B$ & Hammar et al. (1996) \\
\hline pTrc99A & Expression vector & Pharmacia Biotech \\
\hline pMC1 & $\operatorname{csg} G$ cloned into pTrc99A & Chapman et al. (2002) \\
\hline pLR15 & csgG amplified from pMC1 with Trc5F and LR15R cloned into pTrc99A & This study \\
\hline pLR135 & csgE amplified with LR135F and LR135R and cloned into Kpnl/Pstl sites of pLR15 & This study \\
\hline pLR16 & $\begin{array}{l}\text { DNA fragment encoding the PhoA signal sequence amplified with PhoSSF and } \\
\text { LR16R1 cloned into the Ncol/BamHI sites of pTrc99A; sequence encoding } \\
\text { mature CsgG with C-terminal } 6 \text { hi epitope amplified with LR16F2 and GhisR and } \\
\text { cloned into BamHI/Pstl sites of pTrc99A }\end{array}$ & This study \\
\hline pCsgE & csgE amplified with PEF and PER and cloned into the Ncol/BamHI sites of pTrc99A & This study \\
\hline pMC5 & csgEFG cloned into pTrc99A & Chapman et al. (2002) \\
\hline pMC2 & csgG-6his amplified with $\operatorname{Trc5F}$ and GhisR and cloned into pTrc99A & This study \\
\hline pLomp4 & $\begin{array}{l}\text { DNA fragment encoding the signal sequence of } E \text {. coli Lpp fused to residues } \\
41-159 \text { of OmpA cloned into the Ncol/BamHI sites of pTrc99A }\end{array}$ & This study \\
\hline pBAD33 & Expression vector & Guzman et al. (1995) \\
\hline pLR29 & $\begin{array}{l}\text { csgEFG amplified from pMC5 using LR29F and LR29R and cloned into the } \\
\text { Kpnl/Pstl sites of pBAD33. }\end{array}$ & This study \\
\hline pLR46 & $\begin{array}{l}\text { DNA fragment encoding the N-terminus of PapD amplified with LR46F and LR46R } \\
\text { cloned into the Kpnl/Bgl2 sites of pLR29 }\end{array}$ & This study \\
\hline pLR60 & $\begin{array}{l}\text { CmR gene lacking an Ncol site amplified from pBAD33 with LR60F and LR60R and } \\
\text { cloned into Msc1/Sca1 of pLR46 }\end{array}$ & This study \\
\hline pLR92 & $\begin{array}{l}\text { csgG-HA amplified from pMC1 with LR92F and LR92R and cloned into the Sacl/Pstl } \\
\text { sites of pLR60 }\end{array}$ & This study \\
\hline pLR169 & $\begin{array}{l}\text { DNA fragment encoding N-terminal AU-tagged CsgE subcloned into Ncol/Pstl sites } \\
\text { of pLR92 from similarly digested pLR167 }\end{array}$ & This study \\
\hline pLR58 & $\begin{array}{l}\text { csgF-HA amplified with Trc5F and LR58R and cloned into the Ncol/Pstl sites of } \\
\text { pLR92 }\end{array}$ & This study \\
\hline pLR134 & $\begin{array}{l}\text { DNA fragment encoding the first } 42 \text { amino acids of CsgA, containing the s signal } \\
\text { sequence and the first } 22 \text { residues of the mature protein, amplified with LR134F } \\
\text { and LR134R cloned into the Ncol/Pstl sites of pLR92 }\end{array}$ & This study \\
\hline pAph2 & $\begin{array}{l}\text { DNA sequence encoding mature PhoA amended with a C-terminal HA tag amplified } \\
\text { with Aph2F and PhoHAR cloned into the Ncol/Pstl sites of pLR134. The resulting } \\
\text { fusion encodes the first } 42 \text { amino acids of CsgA and the mature PhoA protein }\end{array}$ & This study \\
\hline pAph1 & $\begin{array}{l}\text { phoA-HA amplified with Aph1F and PhoHAR and cloned into the Sacl/Pstl sites of } \\
\text { pLR92 }\end{array}$ & This study \\
\hline pACYC177 & Cloning vector & New England Biolabs \\
\hline pLR1 & $\begin{array}{l}\operatorname{csgBA} \text { promoter amplified with LR1F and LR1R and cloned into the BamHI/Pstl } \\
\text { sites of pACYC177. }\end{array}$ & This study \\
\hline pLR2 & The Ncol/Pstl fragment of pLomp4 subcloned into pLR1 & This study \\
\hline pLR7 & $\begin{array}{l}\text { csgGss-HA amplified from pLR16 with PhoSSF and LR7R and cloned into the } \\
\text { Ncol/Pstl sites of pLR1. }\end{array}$ & This study \\
\hline pLR167 & $\begin{array}{l}\text { DNA fragment encoding N-terminal AU-tagged CsgE amplified with LR169F and } \\
\text { Trc3R and cloned into the BamHI/Pstl sites of pLR7 }\end{array}$ & This study \\
\hline pLR95 & $\begin{array}{l}\text { The Ncol/Pstl fragment of pLR16 containing the csgGss chimera subcloned into } \\
\text { pLR1 }\end{array}$ & This study \\
\hline pLR93 & The Ncol/Pstl of pMC1 containing $\operatorname{csg} G$ subcloned into pLR1 & This study \\
\hline pMC3 & $\operatorname{csg} A-6 h i s$ cloned into pHL3 & Chapman et al. (2002) \\
\hline
\end{tabular}

rocking $1 \mathrm{~h}$ at $4^{\circ} \mathrm{C}$. Twenty-five microlitres of Protein A-agarose bead slurry (Sigma, St Louis, MO) was added and the mixture rocked $1 \mathrm{~h}$ at $4^{\circ} \mathrm{C}$. The beads were pelleted and washed three times in HNE before resuspension in $50 \mu \mathrm{l} 2 \times$ SDS loading buffer.

\section{Antibiotic sensitivity assays}

Plasmid pTRC99A (empty vector) or pMC1 (Ptrc-csgG) were transformed into LSR12 (MC4100:: $\triangle \operatorname{csg} D E F G ; \Delta c s g B A$ ). Strains were grown to stationary phase in LB, diluted 1:100 in fresh media, and grown with agitation for $30 \mathrm{~min}$. At this point, $0.05 \mathrm{mM}$ IPTG was added to the cultures, and bacteria were grown another 30 min before addition of erythromycin or vancomycin. Antibiotic addition was designated as time zero and $\mathrm{OD}_{600}$ measured every $30 \mathrm{~min}$ for $300 \mathrm{~min}$. The $\mathrm{OD}_{600}$ was measured using a Molecular Dynamics UV-Vis spectrophometer. 


\section{Acknowledgements}

We thank Michelle Barnhart and Bob Bender for critically reading the manuscript and John Heuser for the electron micrographs of purified CsgG. This work was supported by NIH award number K22A1054967-01 to M.R.C, Al48689 to S. J. H and \#5-T32-GM07544 awarded to E.M.A.

\section{References}

Augustus, A.M., Celaya, T., Husain, F., Humbard, M., and Misra, R. (2004) Antibiotic-sensitive TolC mutants and their suppressors. J Bacteriol 186: 1851-1860.

Ben Nasr, A., Olsen, A., Sjobring, U., Muller-Esterl, W., and Bjorck, L. (1996) Assembly of human contact phase proteins and release of bradykinin at the surface of curliexpressing Escherichia coli. Mol Microbiol 20: 927-935.

Bian, Z., and Normark, S. (1997) Nucleator function of CsgB for the assembly of adhesive surface organelles in Escherichia coli. EMBO J 16: 5827-5836.

Bose, N., and Taylor, R.K. (2005) Identification of a TcpCTcpQ outer membrane complex involved in the biogenesis of the toxin-coregulated pilus of Vibrio cholerae. J Bacteriol 187: 2225-2232.

Brok, R., Van Gelder, P., Winterhalter, M., Ziese, U., Koster, A.J., de Cock, H., et al. (1999) The C-terminal domain of the Pseudomonas secretin $\mathrm{XcpQ}$ forms oligomeric rings with pore activity. $J$ Mol Biol 294: 1169-1179.

Campbell, A. (1961) Sensitive mutants of bacteriophage lambda. Virology 14: 22-32.

Casadaban, M.J. (1976) Transposition and fusion of the lac genes to selected promoters in Escherichia coli using bacteriophage lambda and Mu. J Mol Biol 104: 541-555.

Chapman, M.R., Robinson, L.S., Pinkner, J.S., Roth, R., Heuser, J., Hammar, M., et al. (2002) Role of Escherichia coli curli operons in directing amyloid fiber formation. Science 295: 851-855.

Chirwa, N.T., and Herrington, M.B. (2003) CsgD, a regulator of curli and cellulose synthesis, also regulates serine hydroxymethyltransferase synthesis in Escherichia coli K12. Microbiology 149: 525-535.

Collinson, S.K., Doig, P.C., Doran, J.L., Clouthier, S., Trust, T.J., and Kay, W.W. (1993) Thin, aggregative fimbriae mediate binding of Salmonella enteritidis to fibronectin. $J$ Bacteriol 175: 12-18.

Collinson, S.K., Parker, J.M., Hodges, R.S., and Kay, W.W. (1999) Structural predictions of AgfA, the insoluble fimbrial subunit of Salmonella thin aggregative fimbriae. $\mathrm{J} \mathrm{Mol} \mathrm{Biol}$ 290: 741-756.

Gerstel, U., Park, C., and Romling, U. (2003) Complex regulation of $\operatorname{csg} D$ promoter activity by global regulatory proteins. Mol Microbiol 49: 639-654.

Guzman, L.M., Belin, D., Carson, M.J., and Beckwith, J. (1995) Tight regulation, modulation, and high-level expression by vectors containing the arabinose PBAD promoter. J Bacteriol 177: 4121-4130.

Hammar, M., Arnqvist, A., Bian, Z., Olsen, A., and Normark, S. (1995) Expression of two csg operons is required for production of fibronectin- and congo red-binding curli polymers in Escherichia coli K-12. Mol Microbiol 18: 661670.
Hammar, M., Bian, Z., and Normark, S. (1996) Nucleatordependent intercellular assembly of adhesive curli organelles in Escherichia coli. Proc Natl Acad Sci USA 93: 6562-6566.

Hardie, K.R., Seydel, A., Guilvout, I., and Pugsley, A.P. (1996) The secretin-specific, chaperon-like protein of the general secretory pathway: separation of proteolytic protection and piloting functions. Mol Microbiol 22: 967-976.

Hung, D.L., Raivio, T.L., Jones, C.H., Silhavy, T.J., and Hultgren, S.J. (2001) Cpx signaling pathway monitors biogenesis and affects assembly and expression of $\mathrm{P}$ pili. EMBO J 20: 1508-1518.

Kayed, R., Head, E., Thompson, J.L., McIntire, T.M., Milton, S.C., Cotman, C.W., and Glabe, C.G. (2003) Common structure of soluble amyloid oligomers implies common mechanism of pathogenesis. Science 300: 486-489.

Lashuel, H.A., Hartley, D., Petre, B.M., Walz, T., and Lansbury, P.T., Jr. (2002) Neurodegenerative disease: amyloid pores from pathogenic mutations. Nature 418: 291.

Loferer, H., Hammar, M., and Normark, S. (1997) Availability of the fibre subunit CsgA and the nucleator protein CsgB during assembly of fibronectin-binding curli is limited by the intracellular concentration of the novel lipoprotein CsgG. Mol Microbiol 26: 11-23.

Mundy, R., Pickard, D., Wilson, R.K., Simmons, C.P., Dougan, G., and Frankel, G. (2003) Identification of a novel type IV pilus gene cluster required for gastrointestinal colonization of Citrobacter rodentium. Mol Microbiol 48: 795809.

Narita, S., Matsuyama, S., and Tokuda, H. (2004) Lipoprotein trafficking in Escherichia coli. Arch Microbiol 182: 1-6.

Nevesinjac, A.Z., and Raivio, T.L. (2005) The Cpx envelope stress response affects expression of the type IV bundleforming pili of enteropathogenic Escherichia coli. $J$ Bacteriol 187: 672-686.

Olsen, A., Jonsson, A., and Normark, S. (1989) Fibronectin binding mediated by a novel class of surface organelles on Escherichia coli. Nature 338: 652-655.

Prigent-Combaret, C., Brombacher, E., Vidal, O., Ambert, A., Lejeune, P., Landini, P., and Dorel, C. (2001) Complex regulatory network controls initial adhesion and biofilm formation in Escherichia coli via regulation of the $\operatorname{csg} D$ gene. J Bacteriol 183: 7213-7223.

Ramer, S.W., Bieber, D., and Schoolnik, G.K. (1996) BfpB, an outer membrane lipoprotein required for the biogenesis of bundle-forming pili in enteropathogenic Escherichia coli. J Bacteriol 178: 6555-6563.

Romling, U., Bian, Z., Hammar, M., Sierralta, W.D., and Normark, S. (1998a) Curli fibers are highly conserved between Salmonella typhimurium and Escherichia coli with respect to operon structure and regulation. J Bacteriol 180: 722731.

Romling, U., Sierralta, W.D., Eriksson, K., and Normark, S. (1998b) Multicellular and aggregative behaviour of Salmonella typhimurium strains is controlled by mutations in the agfD promoter. Mol Microbiol 28: 249-264.

Ruiz, N., and Silhavy, T.J. (2005) Sensing external stress: watchdogs of the Escherichia coli cell envelope. Curr Opin Microbiol 8: 122-126.

Schmidt, S.A., Bieber, D., Ramer, S.W., Hwang, J., Wu, C.Y., and Schoolnik, G. (2001) Structure-function analysis of 
$\mathrm{BfpB}$, a secretin-like protein encoded by the bundle-forming-pilus operon of enteropathogenic Escherichia coli. $J$ Bacteriol 183: 4848-4859.

Sjobring, U., Pohl, G., and Olsen, A. (1994) Plasminogen, absorbed by Escherichia coli expressing curli or by Salmonella enteritidis expressing thin aggregative fimbriae, can be activated by simultaneously captured tissue-type plasminogen activator (t-PA). Mol Microbiol 14: 443-452.

Thanassi, D.G., and Hultgren, S.J. (2000) Assembly of complex organelles: pilus biogenesis in Gram-negative bacteria as a model system. Methods 20: 111-126.

Thanassi, D.G., Saulino, E.T., Lombardo, M.J., Roth, R., Heuser, J., and Hultgren, S.J. (1998) The PapC usher forms an oligomeric channel: implications for pilus biogenesis across the outer membrane. Proc Natl Acad Sci USA 95: 3146-3151.

Zogaj, X., Nimtz, M., Rohde, M., Bokranz, W., and Romling, U. (2001) The multicellular morphotypes of Salmonella typhimurium and Escherichia coli produce cellulose as the second component of the extracellular matrix. Mol Microbiol 39: 1452-1463.

Zogaj, X., Bokranz, W., Nimtz, M., and Romling, U. (2003) Production of cellulose and curli fimbriae by members of the family Enterobacteriaceae isolated from the human gastrointestinal tract. Infect Immun 71: 4151-4158.

\section{Supplementary material}

The following supplementary material is available for this article online:

Table S1. Oligonucleotide primers used in this study. Lower case letters indicate sequences that do not hybridize to the template used in the PCR reaction.

This material is available as part of the online article from http://www.blackwell-synergy.com 